\title{
Effect of an acute dose of omega-3 fish oil following exercise-induced muscle damage
}

Jakeman, J. R.

Lambrick, D. M.

Wooley, B.

Babraj, John A.

Faulkner, J. A.

The final publication is available at Springer via

https://dx.doi.org/10.1007/s00421-017-3543-y 
Effect of an acute dose of omega-3 fish oil following exercise-induced muscle damage

J.R. Jakeman ${ }^{1}$, D.M. Lambrick ${ }^{2}$, B. Wooley ${ }^{3}$, J.A. Babraj ${ }^{4}$, J.A. Faulkner ${ }^{5}$

${ }^{1}$ Department of Sport and Health Science, Oxford Brookes University, Oxford, OX3 OBP, UK

${ }^{2}$ Faculty of Health Sciences, University of Southampton, Southampton, SO17 1BJ, UK

${ }^{3}$ School of Sport and Exercise, Massey University, Wellington, 6140, New Zealand

${ }^{4}$ Division of Sport and Exercise Science, University of Abertay, Dundee, DD1 1HG, UK

${ }^{5}$ Department of Sport and Exercise, University of Winchester, Winchester SO22 4NR, UK 
Abstract

Purpose The purpose of this double-blind, placebocontrolled study was to examine the effect of two fish oil supplements, one high in EPA (750 mg EPA, $50 \mathrm{mg}$ DHA) and one low in EPA (150 mg EPA, 100 $\mathrm{mg} \mathrm{DHA})$, taken acutely as a recovery strategy following EIMD.

Methods Twenty-seven physically active males ( $26 \pm 4$ year, $1.77 \pm 0.07 \mathrm{~m}, 80 \pm 10 \mathrm{~kg}$ ) completed 100 plyometric drop jumps to induce muscle damage. Perceptual (perceived soreness) and functional (isokinetic muscle strength at $60^{\circ}$ and $180^{\circ} \mathrm{s}^{-1}$, squat jump performance and countermovement jump performance) indices of EIMD were recorded before, and 1, 24, 48, 72, and $96 \mathrm{~h}$ after the damaging protocol. Immediately after the damaging protocol, volunteers ingested either a placebo (Con), a low-EP fish oil (Low EPA) or a high-EPA fish oil (High EPA) at a dose of $1 \mathrm{~g}$ per $10 \mathrm{~kg}$ body mass.

Results A significant group main effect was observed for squat jump, with the High EPA group performing better than Con and Low EPA groups (average performance decrement, 2.1, 8.3 and $9.8 \%$, respectively), and similar findings were observed for countermovement jump performance, (average performance decrement, 1.7, 6.8 and $6.8 \%$, respectively, $p=0.07$ ). Significant time, but no interaction main effects were observed for all functional and perceptual indices measured, although large effect sizes demonstrate a possible ameliorating effect of high dose of EPA fish supplementation (effect sizes $\geq 0.14$ ).

Conclusion This study indicates that an acute dose of high-EPA fish oil may ameliorate the functional changes following EIMD.

Keywords: Omega-3, Recovery, Athlete, Training, Nutrition 


\section{Introduction}

Exercise-induced muscle damage (EIMD) is a well-recognised and documented condition, and primarily occurs as a result of unaccustomed or eccentrically biased exercise (Proske and Morgan 2001). EIMD manifests through increases in localised inflammation, decreases in functional muscle capability and, most noticeably, through increases in perceived muscle soreness $24-72 \mathrm{~h}$ following a damaging bout of exercise (Jakeman et al. 2009; Webb and Willems2010). These transient symptoms can be relatively debilitating, and while there may be positive long-term adaptations elicited as a result of EIMD, those involved in the preparation of athletes for competition, in particular multiple competitions in a short time frame, have sought methods of facilitating recovery to expedite a return to optimal performance. To this end, methods such as cold-water immersion, massage, and active recovery have been considered (Barnett 2006), although the evidence advocating the use of these treatments remains equivocal (Bailey et al. 2007; Jakeman et al. 2009; Hilbert et al. 2003). In addition, it has been demonstrated that exercise which results in muscle damage can confer a protective effect on an individual, known as the repeated bout effect, reducing symptoms from similar future exercise as long as the exercise bouts in each condition are similar (Margaritelis et al. 2015).

While physical therapies have shown some success in alleviating the symptoms of EIMD, nutritional supplementation is also widely used to facilitate recovery following EIMD, with a number of studies considering the efficacy of non-steroidal anti-inflammatory drugs for example (Bourgeois et al. 1999; Paoloni et al. 2009). Omega-3 fish oil is a supplement which has increased in popularity as it has potent anti-inflammatory effects and is associated with a lower risk of coronary heart disease (Hu et al. 2002). The two primary constituents of fish oil, eicosapentaenoic acid (EPA) and docosahexaenoic acid (DHA), are thought to reduce the production and release of arachidonic acid and proinflammatory prostaglandins, among others, with this being particularly true of EPA (Tartibian et al. 2011; Lenn et al. 2002). Accordingly, it has been suggested that fish oil can ameliorate the symptoms of EIMD, including soreness and swelling (Jouris et al. 2011). Typically, prolonged supplementation periods, including 7, 21, 26 and 30 days, have been used to assess the effect of fish oil in moderating EIMD, as it has been demonstrated that chronic supplementation can result in EPA and DHA being incorporated into cellular membranes (Jouris et al. 2011; Houghton and Onambele 2012; Mickleborough et al. 2015; Lembke et al. 2014; Lenn et al. 2002). Recent research has concluded that EPA and DHA supplementation in a 2:1 ratio may be beneficial to attenuating symptoms of EIMD when consumed chronically ( 8 weeks; Tsuchiya et al. 2016). While these findings are of practical interest, anecdotal evidence suggests that individuals consume fish oil immediately 
postexercise in an attempt to ameliorate symptoms of EIMD. There is currently little evidence to support this approach. As such, research into the effects of acute supplementation, in respect to real-world practices, is warranted, particularly to guide the athletes and coaches intending to maximize recovery following strenuous exercise.

The purpose of this study was, therefore, to examine the efficacy of an acute dose of omega- 3 fish oil on recovery following EIMD. It was hypothesised that an acute dose of omega-3 fish oil would aid recovery following EIMD, and that a high dose of EPA would have a greater effect than a low dose of EPA.

\section{Methods}

Participants

For this double-blind, placebo-controlled study, 27 physically active ( $>3 \mathrm{~h} \mathrm{wk}^{-1}$ of vigorous athletic training involving repeated bouts of high-intensity intermittent training) men volunteered to participate ( $26 \pm 4$ year, $1.77 \pm 0.07 \mathrm{~m}, 80 \pm 10 \mathrm{~kg}$ ). Participants were injury-free, healthy and asymptomatic of any illness, as confirmed through health screening procedures (ACSM 2013), and were excluded if they had engaged in specific lower limb eccentric or plyometric training in the previous 6 months. Volunteers were asked to refrain from any exhausting physical activity or from using any additional form of recovery method (e.g., ice, massage, nutritional supplements) in an attempt to attenuate the negative effects of EIMD during their participation in this study. The study was conducted in agreement with the policies and guidelines of the institutional Human Ethics Committee, and informed consent was obtained from all individual participants included in the study. A priori calculations of statistical power indicated that this sample size was appropriate to satisfy power at or above $80 \%$ (Jakeman et al. 2010).

Procedures

Following the collection of anthropometric data, participants completed an exercise protocol designed to induce muscle damage. Following this, participants were randomly assigned to one of the three groups: High EPA ( $n=9$; age $25.5 \pm 5.2$ years, height $1.74 \pm 0.06 \mathrm{~m}$, weight $76.5 \pm 12.6 \mathrm{~kg}$ ), Low EPA ( $n=9$; age $25.6 \pm 4.8$ years, height $1.82 \pm 0.09 \mathrm{~m}$, weight $80.2 \pm 12.0 \mathrm{~kg}$ ) or placebo $(n=9$; age $26.2 \pm 4.2$ years, height $1.78 \pm 0.01 \mathrm{~m}$, weight $82.9 \pm 12.1 \mathrm{~kg}$ ). Physiological and perceptual characteristics were assessed in the following sequential order, prior to- [baseline (BL)] and following $(1,24,48,72$ and $96 \mathrm{~h})$ the EIMD protocol: myofibre protein concentration [plasma creatine kinase (CK)], inflammatory markers [Interleukin 6 (IL-6)], perceived muscle soreness, countermovement and squat jump height and isokinetic muscle strength (maximal knee extension). Data were collected $1 \mathrm{~h}$ 
after damaging exercise to provide an indicator of the magnitude of immediate muscle damage, without the confounding factor of acute fatigue resultant from the muscle-damaging protocol.

Plyometric exercise protocol

To induce muscle damage, participants performed $10 \times 10$ repetitions of plyometric drop jumps, with 10 s separating each jump, and 60-s recovery between sets, beginning after completion of the tenth jump of each set. Under the guidance of an experienced strength and conditioning coach, participants were asked to step from a 0.6-m-high box, land (with both feet) in a squat position, with approximately $90^{\circ}$ of hip and knee flexion, and then perform a maximal vertical jump in a continuous movement. This protocol has been used successfully elsewhere (Highton et al. 2009).

\section{Randomisation}

On completion of the plyometric exercise protocol, participants were randomised into one of three groups: Con-participants received placebo capsules which consisted of filler oil, flavour masker and gelatine; High EPA-participants consumed $1 \mathrm{~g} 10 \mathrm{~kg}$ body mass of fish oil capsules; EPA $750 \mathrm{mg}$, DHA $50 \mathrm{mg}$; Low EPA-participants consumed 1g $10 \mathrm{~kg}$ body mass of fish oil capsules; EPA $150 \mathrm{mg}$, DHA $100 \mathrm{mg}$. All supplements were provided in 1-g capsules, and given to participants in one dose upon completion of the plyometric protocol. Allocation to groups was by means of sealed envelopes drawn by the participants, designed for an equal allocation between the three groups. A researcher who was not involved in data collection provided capsules, while data analysts were blinded from the grouping code.

\section{Assessment of EIMD}

Jump performance

Squat and countermovement jump height was assessed using a vaned jump and reach apparatus, which provides the maximum height reached to the nearest centimetre (Yardstick, Swift Systems, Lismore, Australia), with the best of three attempts being recorded in accordance with previous research (Ali et al. 2011; Pournot et al. 2011). Each jump was completed after verbal command and demonstration, with a minimum of one-minute between jumps, following the protocol of Jakeman et al. (2010). Participants were given time prior to beginning the experimental procedure to familiarise themselves with this apparatus and jump procedures. Typical CV\% for these measures within our lab is $3-3.5 \%$ for these jumps.

Isokinetic muscle strength 
Dynamic muscle function was assessed using isokinetic dynamometry. Participants were seated upright with the upper body and experimental leg secured to reduce extraneous movement. The axis of rotation of the knee of the dominant leg was aligned with the dynamometer which was maintained throughout the testing period. Each participant's body position was recorded and replicated for subsequent tests. Participants completed five maximal voluntary isokinetic concentric contractions at $60^{\circ}$ and $180^{\circ} \mathrm{s}^{-1}$ through approximately a $90^{\circ}$ range of movement from $90^{\circ}$ knee flexion on the dominant leg. The best of five maximal gravity-corrected concentric contractions (Biodex 3 Medical Systems, New York, USA) of the knee extensors was taken as the criterion measure of muscle strength.

Perceived muscle soreness

Perceived soreness was assessed using a 10-cm visual analogue scale, with 0 (no pain) and 10 (worst pain ever) at the two extremes. Participants were instructed to assume an unweighted squat of approximately $90^{\circ}$ and mark their perceived soreness on a horizontal line between 0 and 10 (Twist and Eston 2005).

Blood markers

A 6-ml venous blood sample was drawn from the antecubital vein at each time point, and was centrifuged (Medifuge, Heraeus Sepatech, Germany) for 10 min to obtain plasma samples (Woolley et al., 2014), which were frozen at $-80^{\circ} \mathrm{C}$ until analysis. Automated spectrophotometry (Cobas 6000 , Roche Diagnostics, Indianapolis, USA) was used to analyse samples for plasma CK concentration, and an ELISA kit (Enzyme-Linked Immuno-Sorbent Assays, Enzo Life Sciences, New York, USA) with a sensitivity of $6.01 \mathrm{pg} \cdot \mathrm{ml}$ and specific for bioactive human IL-6 was used to measure interleukin-6.

Statistical analysis

Data for creatine kinase and IL- 6 were log-transformed to address assumptions of sphericity, and all performance data were analysed as a proportion of baseline (\%). A series of two-factor repeatedmeasures analysis of variance; Group (High EPA, Low EPA, Con) by Time (BL, 1, 24, 48, 72, and $96 \mathrm{~h}$ ) were used to analyse data. The Mauchly sphericity test was used to test assumptions of homogeneity of variance. If this was violated, the Greenhouse-Geisser value was used to adjust degrees of freedom to increase the critical value of the $F$ ratio. Effect sizes were reported as partial eta-squared ( $\eta 2 p)$, whereby $0.01,0.06$ and 0.14 represented a small, medium and large effect (Cohen 1988). Alpha was set at 0.05 throughout all analyses, and all data were analysed using the statistical package SPSS for Windows, PC 


\section{Results}

Jump performance

A significant main effect for Time was observed for both squat jump (F3,77 =14.4, $p<0.001, \eta 2 p=$ 0.38 ) and countermovement jump performance $(F 2.9,70=10.5, p<0.05, \eta 2 p=0.31)$, whereby the significant decreases in performance which were observed at $1 \mathrm{~h}$ were maintained until $96 \mathrm{~h}$ postEIMD (Fig. 1). A significant Group effect was also observed for Squat jump performance ( $F 2,24=7.5$, $p<0.05, \eta 2 p=0.39$ ), with the High EPA group performing significantly better than either the Low EPA or Con groups. Although non-significant, a similar trend was observed for countermovement jump performance $(F 2,24=3.19, p=0.07, \eta 2 p=0.2)$. There was no Group $x$ Time interaction for either squat- or countermovement jump performance ( $p>0.05)$; however, a large effect was observed (both eta $=0.13$ ).

Isokinetic muscle strength

A significant Time main effect was observed for knee extensor strength at both $60^{\circ} \mathrm{s}-1(\mathrm{~F} 5,120=$ 25.7, $p<0.05, \eta 2 p=0.52)$ and $180^{\circ} s-1(F 5,120 p<0.05, \eta 2 p=0.42$; Fig. 2). There was a significant reduction in strength at $1 \mathrm{~h}$ at both $60^{\circ}$ and $180^{\circ} \mathrm{s}-1$, which persisted for 96 and $72 \mathrm{~h}$, respectively, following damaging exercise. There were no differences between Groups $(F 2,24=1.9, p>0.05, \eta 2 p$ $=0.14 ; F 2,24=0.4, p>0.05, \eta 2 p=0.31$ for $60^{\circ}$ and $180^{\circ} s-1$, respectively), nor any Group by Time interactions $\left(F 10,120=1.5, p>0.05, \eta 2 p=0.11\right.$ and $F 10,120=0.62, p>0.05, \eta 2 p=0.05$ for $60^{\circ}$ and $180^{\circ} \mathrm{s}-1$, respectively).

Perceived muscle soreness

There was a significant main effect for Time ( $F 2.5,60.5=36.6, p<0.05, \eta 2 p=0.60)$, but no Group $(F 2,24=0.69, p>0.05, \eta 2 p=0.05)$ or Group $\times$ Time interaction (F5, 60.5 = 1.05, p>0.05, $\eta 2 p=$ 0.08). In all Groups, perceived soreness peaked at $24 \mathrm{~h}$ following EIMD, and recovered to baseline values at $96 \mathrm{~h}$ (Fig. 3).

Myofibre proteins

There was a significant Time main effect for creatine kinase concentration (F1.6,36 $=13.9, p<0.05$, $\eta 2 p=0.38)$, but no Group or Group $\times$ Time interaction $(F 2,22=1.6, p>0.05, \eta 2 p=0.12$ and F3.2,36 $=0.143, p>0.05, \eta 2 p=0.13$, respectively). There were no Time $(F 2.2,41.8=1.04, p>0.05, \eta 2 p=$ $0.052)$, Group (F2,22 $=0.225, p>0.05, \eta 2 p=0.12)$ or Group $\times$ Time interaction effects $(F 3.2,36=$ $0.143, p>0.05, \eta 2 p=0.013$ ) for IL-6 following EIMD (Table 1). 


\section{Discussion}

This study assessed whether an acute dose of omega-3 fish oil immediately following strenuous, eccentrically biased exercise would moderate symptoms of EIMD. The current study shows that acute ingestion of omega-3 fish oil, in particular a supplement which is high in EPA, is beneficial to some markers of exercise performance following EIMD, in comparison with either a low-dose EPA supplement, or a placebo. While many studies have investigated the efficacy of omega-3 fish oil as a recovery strategy following EIMD, to our knowledge, this is the first study to consider a large, acute dose of omega-3 in such a manner. To facilitate the practical applicability of the current work and descriptive usefulness, we have included both effect sizes and confidence intervals relating to the collected data (Richardson 2011). While statistical significance is important, in studies with small sample sizes such as those often seen in the sport and exercise science area, consideration of the magnitude of effect is sometimes more useful for the full evaluation of data (Rhea 2004).

It is well established that physical performance is detrimentally effected by EIMD, with a number of studies showing reductions in peak power generation, jump performance and time trial exercise (Byrne and Eston 2002; Twist and Eston 2005; Burt and Twist 2011). Consistent with our hypothesis as indicated in Fig. 1a, and when observing a large effect size ( $\eta 2 p=0.39)$, high-dose EPA supplementation attenuated the decrement in average squat jump performance $(-2.1 \%)$ in comparison to both low-dose EPA (-9.8\%) and placebo (-8.3\%) supplementation. Importantly, participants in the High EPA group had recovered to within $\sim 2 \%$ of baseline performance at $24 \mathrm{~h}$, whereas participants in the Low EPA or Con groups remained $\sim 12 \%$ lower than baseline performance at $24 \mathrm{~h}$. From a practical perspective, the impairment in squat jump performance at $1 \mathrm{~h}$ in the High EPA group was equivalent to the impairment observed at $96 \mathrm{~h}$ for both the Low EPA and placebo groups, indicating a faster recovery of performance capability. A similar pattern was also observed with countermovement jump performance (average decrement of 1.7, 6.8, and 6.8\% in High EPA, Low EPA and Con, respectively; $\eta 2 p=0.2$ ). For example, the decrement in performance was only $4 \%$ after $1 \mathrm{~h}$ for the High EPA group in comparison with the 10.1 and 15\% decrements observed for the Con or Low EPA groups, respectively. Similar to the changes in squat jump performance, the High EPA group returned to within $2 \%$ of baseline performance after $24 \mathrm{~h}$, in comparison with the Control and Low groups, whereby there countermovement jump performance was still 4.4 and $3.8 \%$ below baseline at their $96 \mathrm{~h}$ re-assessment (Fig. 1b).

It is widely agreed that one of the initial consequences of EIMD is harm to the contractile machinery of the muscle, manifest by the disruption of sarcomeres, which is thought to prevent the muscle from contracting normally (Proske and Morgan 2001). Squat jump exercise relies heavily on muscle 
contractile properties by limiting as much as possible, the influence of the stretch-shortening cycle. Therefore, reductions in squat jump performance are likely to be related to an inability to contract musculature due to the bout of damaging exercise. Reductions in contractile force capability are compounded by the possibility that voluntary muscle activation is negatively affected by EIMD (Prasartwuth et al. 2005). However, Prasartwuth et al. (2005) suggest that reductions in maximal voluntary muscle activation may be a partial cause of initial force loss. The countermovement jump in this case relies on the influence of the stretch-shortening cycle, as well as the contractile machinery, and therefore, changes in neural factors are likely to have a compounding effect on performance. With the current data showing an immediate protective effect in the High group, it is, therefore, possible that supplementation allowed for greater voluntary activation, a suggestion supported by Lewis et al. (2015). In their study, although their findings were not in relation to EIMD, Lewis and colleagues reported that peripheral neuromuscular function may be improved in experimental athletic situations as a result of consumption of omega-3.

When considering the isokinetic muscle function at $60^{\circ}$ and $180^{\circ} \mathrm{s}^{-1}$, despite no statistical differences between groups, large effect sizes were noted ( 0.14 and 0.31 , respectively). Average performance changes at $60^{\circ}$ and $180^{\circ} \mathrm{s}^{-1}$ were similar to changes in the squat and countermovement jump, respectively, and may reflect similar speed characteristics between these measures. Again, changes in performance at $60^{\circ} \mathrm{s}^{-1}$ are likely to be due to a reduced ability of the contractile machinery used at this relatively slow movement speed. The pattern of change in performance at $180^{\circ}$ was similar in all groups, and while there was no statistically significant difference in group, there is a trend toward a protective effect at $60^{\circ} \mathrm{s}^{-1}$. The large effect sizes, here, indicate that for 72 $\mathrm{h}$ after damaging exercise, individuals in the High group seemed to experience a lower reduction in performance (Fig. 2). It is worth noting that isokinetic muscle function was analysed concentrically, to be more relevant to the prime mover muscles involved in many sporting activities, such as running and jumping (McBride et al. 2008). However, it is possible that the eccentric damaging protocol would have been more pronounced on an eccentric isokinetic movement pattern, and given that eccentric muscle movements are imperative for movement stability (LeStayo et al. 2003), future studies may find it interesting to consider eccentric muscle function assessment, in place of or in addition to concentric muscle function assessment, particularly where injury is a primary topic of interest.

One of the most noticeable effects of EIMD is delayed onset muscle soreness (DOMS), which may be associated with an increase in swelling resulting from the bout of damaging exercise (Miller et al. 2004). While this is a normal process of recovery and adaptation within the body, symptoms of EIMD 
such as DOMS are detrimental to performance in athletic situations. While there is benefit to physical activity which results in EIMD (see McHugh 2003 for example), individuals involved in regular and repeated competition in a short time period (i.e., tournament play) may wish to forgo any positive adaptive effects in favour of being able to maintain optimal athletic performance. As a result of studies demonstrating an anti-inflammatory effect of omega-3 (Tartibian et al. 2011; Mickleborough et al. 2015), it was hypothesised that IL-6, and perceived muscle soreness may have been reduced in the High and Low EPA groups. Although not conclusive (Weldon et al. 2007), EPA has been shown to be a more potent inhibitor of the inflammatory response than DHA (Mickleborough et al. 2009), and it may have been expected that this would result in the High EPA group having reduced symptoms of inflammatory markers. Such findings were not evident in the present study as similar perceptions of muscle soreness and inflammatory markers were observed between groups. While this is in contrast to other studies which have considered similar scenarios (Jouris et al. 2011), the lack of impact on perceived muscle soreness reflects the work by Lenn et al. (2002) and Houghton and Onambele (2012). It is likely that the lack of effect in the current work was due to the nature of the supplementation, in the fact that it was an acute, rather than a chronic supplementation protocol. Prolonged ingestion of omega-3 fish oil has been shown to result in changes to muscle cell membrane, altering its permeability and making the cell less susceptible to EIMD. Tartibian et al. (2009), for example, reported reduced perceived soreness following strenuous eccentric exercise in a group supplemented for 30 days with omega-3. The authors suggested that this may be due to a decrease in muscle damage or reduction in inflammation experienced by the supplementing group, in comparison with both a placebo group, and a non-supplementing control, and is a theme which has been reported elsewhere (Tsuchiya et al. 2016). Given that the current study employed an acute dose of omega-3, it may have had little effect on inflammatory markers immediately after damaging exercise, but may have been influential in promoting recovery at later time points. Previous research by Gingras et al. (2007) has indicated that ingestion of omega-3 oils can alter protein synthesis and insulin sensitivity, and positive alterations in anabolic responses have also been reported by Smith et al. (2011a, b) in human models. This suggests that omega-3 fatty acids can stimulate muscle protein synthesis, which may also have influenced the recovery process; however, further study would be required to determine this. Although there were no indications that inflammatory markers had been affected by the treatment, it is worth noting that some inflammatory markers, particularly creatine kinase, relate poorly to the magnitude or time course of EIMD as indicated by other markers (for review, see Warren et al. 1999). While not measured, it is possible that swelling of the affected limbs may have been reduced by the treatment and, therefore, contribute to the differences in the response observed on the performance measures. 
As noted previously, the supplementation strategy used in the current study is very different to that used by others, in that it focuses on an acute, large dose supplementation. Recently, Tsuchiya et al. (2016) investigated the use of a ratio of EPA:DHA of approximately 2:1, with participants ingesting $600 \mathrm{mg}$ EPA and $260 \mathrm{mg}$ DHA each day for 8 weeks. Participants in the current study consumed either 15:1 or 1.5:1 EPA:DHA ratio immediately after EIMD, which was relativised for body mass, an important consideration which has also not been previously made. While it appears that the High group (15:1 EPA:DHA) experienced an enhanced recovery, the mechanisms responsible for this were not investigated in the current study, and should be considered in future work. As a result of the novel dosing strategies (an acute dose relative to body mass), it makes the comparison of the current results with other similar studies problematic, and further research on the areas of dose, duration and body mass/composition should be considered.

Conclusions

In conclusion, this double-blind, placebo-controlled study has demonstrated that a high dose of highEPA omega-3 fish oil immediately following strenuous, eccentrically biased exercise may moderate symptoms of EIMD. While statistically non-significant, the large effect sizes observed suggest that a number of the considered performance measures were positively influenced by the High but not the Low EPA dose. During both the squat and countermovement jump, the performance decrement in those individuals taking the high-dose omega-3 fish oil was significantly attenuated, nearly returning to their baseline level of performance within $24 \mathrm{~h}$. While these findings are of practical interest, further research is needed to determine optimal supplementation strategies, both in terms of duration and dose, as well as the optimal EPA:DHA ratio.

\section{Acknowledgements}

We are grateful to Take Omega 3 for providing the fish oil and placebo supplementation.

\section{Ethical approval}

All procedures performed in studies involving human participants were in accordance with the ethical standards of the institutional and/or national research committee and with the 1964 Helsinki declaration and its later amendments or comparable ethical standards. 


\section{References}

Ali A, Creasy R, Edge J (2011) The effect of graduated compression stockings on running performance. J Strength Cond Res 25:1385-1392

American College of Sports Medicine (2013) ACSM's Guidelines for Exercise Testing and Prescription, 9th edn. Lippincott, Williams \& Wilkins, Philadelphia, PA

Bailey D, Erith S, Griffin P, Dowson A, Brewer D, Gant N, Williams C (2007) Influence of cold-water immersion on indices of muscle damage following prolonged intermittent shuttle running. J Sports Sci 25:1163-1170

Barnett A (2006) Using recovery modalities between training sessions in elite athletes. Sports Med 36:781-796

Bourgeois J, MacDougall D, MacDonald J, Tarnopolsky M (1999) Naproxen does not alter indices of muscle damage in resistance exercise trained men. Med Sci Sports Exerc 31:4-9

Burt D, Twist C (2011) The effects of exercise-induced muscle damage on cycling time-trial performance. J Strength Cond Res 25:2185-2192

Burt D, Lamb K, Nicholas C, Twist C (2013) Effects of repeated bouts of squatting exercise on submaximal endurance running performance. Eur J Appl Physiol 113:285-293

Byrne C, Eston R (2002) The effect of exercise-induced muscle damage on isometric and dynamic knee extensor strength and vertical jump performance. J Sports Sci 20:417-425

Cohen J (1988) Statistical power analysis for the behavioural sciences (2nd Ed.) Laurence Erlbaum Associates, USA

Gingras A-A, White P, Chouinard P, Julien P, Davis T, Dombrowski L, Couture Y, Dubreuil P, Myre A, Bergeron K, Marette A, Thivierge M (2007) Long-chain omega-3 fatty acids regulate bovine wholebody protein metabolism by promoting muscle insulin signaling to the Akt-mTOR-S6K1 pathway and insulin sensitivity. J Physiol 579:269-284

Highton J, Twist C, Eston R (2009) The effects of exercise-induced muscle damage on agility and sprint running performance. J Exerc Sci Fit 7:24-30

Hilbert J, Sforzo G, Swensen T (2003) The effects of massage on delayed onset muscle soreness. Br J Sports Med 37:72-75 
Houghton D, Onambele G (2012) Can a standard dose of eicosapentaenoic acid (EPA) supplementation reduce the symptoms of delayed onset muscle soreness? J Int Soc Sports Nut 9:2 Hu F, Bronner L, Willett W, Stampfer M, Rexrode K, Albert C, Hunter D, Manson J (2002) Fish and Omega-3 fatty acid intake and risk of coronary heart disease in women. J American Medical Assoc $287: 14$

Jakeman J, Macrae R, Eston R (2009) A single 10-min bout of coldwater immersion therapy after strenuous plyometric exercise has no beneficial effect on recovery from the symptoms of exercise induced muscle damage. Ergonomics 52:456-460

Jakeman J, Byrne C, Eston R (2010) Lower limb compression garment improves recovery from exercise-induced muscle damage in young, active females. Eur J Appl Physiol 109:1137-1144 Jouris K, McDaniel J, Weiss E (2011) The effect of omega-3 fatty acid supplementation on the inflammatory response to eccentric strength exercise. J Sports Sci Med 10:432-438

Lembke P, Capodice J, Hebert K, Swenson T (2014) Influence of omega-3 (N3) index on performance and wellbeing in young adults after heavy eccentric exercise. J Sports Sci Med 13:151-156

Lenn J, Uhl T, Mattacola C, Boissonneault G, Yates J, Ibrahim W, Bruckner G (2002) The effects of fish oil and isoflavones on delayed onset muscle soreness. Med Sci Sport Exerc 34:1605-1613.

LeStayo P, Woolf J, Lewek M, Snyder-Mackler L, Reich T, Lindstedt S (2003) Eccentric muscle contractions: their contribution to injury, prevention, rehabilitation and sport. J Orthop Sports Phys Ther 33:557-571

Lewis E, Radonic P, Wolever T, Wells G (2015) 21 days of mammalian omega-3 fatty acid supplementation improves aspects of neuromuscular function and performance in male athletes compared to olive oil placebo. J Int Soc Sports Nut 12:28

Margaritelis N, Theodorou A, Baltzopoulos V, Maganaris C, Paschalis V, Kyparos A, Nikolaides M (2015) Muscle damage and inflammation after eccentric exercise: can the repeated bout effect be removed? Phys Rep 3(12):e12648. doi:10.14814/phy2.12648

McBride J, McCaulley G, Cormie P (2008) influence of preactvitiy and eccentric muscle activity on concentric performance during vertical jumping. J Strength Cond Res 22:750-757 
McHugh M (2003) Recent advances in the understanding of the repeated bout effect: the protective effect against muscle damage from a single bout of eccentric exercise. Scand J Med Sci Sports 13:8897

Mickleborough T, Tecklenburg S, Montgomery G, Lindley M (2009) Eicosapentaenoic acid is more effective than docosahexaenoic transcription from LPS-induced human asthmatic alveolar macrophage cells. Clin Nut 28:71-77.

Mickleborough T, Sinex J, Platt D, Chapman R, Hirt M (2015) The effects PCSO-524, a patented marine oil lipid and omega-3 PUFA blend derived from the New Zealand green lipped mussel (Perna canaliculus) on indirect markers of muscle damage and inflammation after muscle damaging exercise in untrained men: a randomized, placebo controlled trial. J Int Soc Sports Nut 12:10 Miller P, Bailey S, Barnes M, Derr S, Hall E (2004) The effects of protease supplementation on skeletal muscle function and DOMS following downhill running. J Sports Sci 22:365-372

Paoloni J, Milne C, Orchard J, Hamilton B (2009) Non-steroidal anti-inflammatory drugs in sport medicine: guidelines for practical but sensible use. Br J Sports Med 43:863-865

Pournot H, Bieuzen F, Duffield R, Lepretre P-M, Cozzolino C, Hausswirth C (2011) Short term effects of various water immersions on recovery from exhaustive intermittent exercise. Eur J Appl Physiol 111:1287-1295

Prasartwuth O, Taylor J, Gandevia S (2005) Maximal force, voluntary activation and muscle soreness after eccentric damage to human elbow flexor muscles. J Physiol 567:337-348

Proske U, Morgan D (2001) Muscle damage from eccentric exercise: mechanism, mechanical signs, adaptation and clinical applications. J Physiol 537:333-345

Rhea M (2004) Determining the magnitude of treatment effects in strength training research through the use of the effect size. J Strength Cond Res 18:918-920

Richardson J (2011) Eta squared and partial eta squared as measures of effect size in educational research. Ed Res Rev 6:135-147.

Smith G, Atherton P, Reeds D, Mohammed B, Rankin D, Rennie M, Mittendorfer B (2011a) Dietary omega-3 fatty acid supplementation increases the rate of muscle protein synthesis in older adults: a randomized controlled trial. American J Clin Nut 93:402-412 
Smith G, Atherton P, Reeds D, Mohammed B, Rankin D, Rennie M, Mittendorfer B (2011b) Omega-3 polyunsaturated fatty acids augment the muscle protein anabolic response to hypoaminoacidemiahyperinsulinemia in healthy young and middle aged men and women. Clin Sci 121:267-278

Tartibian B, Maleki B, Abbasi A (2009) The effects of ingestion of omega-3 fatty acids on perceived pain and external symptoms of delayed onset muscle soreness in untrained men. Clin J Sport Med 19:115-119

Tartibian B, Maleki B, Abbasi A (2011) Omega-3 fatty acids supplementation attenuates inflammatory markers after eccentric exercise in untrained men. Clin J. Sports Med 21:131-137 Tsuchiya Y, Yanagimoto K, Nakazato K, Hayamizu K, Ochi E (2016) Eicosapentaenoic and docosahexaenoic acids-rich fish oil supplementation attenuates strength loss and limited joint range of motion after eccentric contractions: a randomized, double-blind, placebo-controlled, parallelgroup trial. Eur J Appl Physiol 116:1179-1188

Twist C, Eston R (2005) The effects of exercise-induced muscle damage on maximal intensity intermittent exercise performance. Eur J Appl Physiol 94:652-658

Warren G, Lowe D, Armstrong R (1999) Measurement tools used in the study of eccentric contraction-induced injury. Sports Med 27:43-59

Webb E, Willems M (2010) Effects of wearing graduated compression garment during eccentric exercise. Med Sport 14:193-198.

Weldon S, Mullen A, Loscher C, Hurley L, Roche H (2007) Docosehexaenoic acid induces an antiinflammatory profile in lipopolysaccharide- stimulated human THP-1 macrophages more effectively than eicosapentaenoic acid. J Nut Biochem 18:250-258 
Table 1 Creatine kinase and IL-6 concentration before and up to $96 \mathrm{~h}$ after damaging exercise

Data presented as mean $( \pm s d)$

\begin{tabular}{|c|c|c|c|c|c|c|c|}
\hline \multicolumn{8}{|l|}{ Time } \\
\hline & Condition & Baseline & $1 \mathrm{~h}$ & $24 \mathrm{~h}$ & $48 \mathrm{~h}$ & $72 \mathrm{~h}$ & $96 \mathrm{~h}$ \\
\hline \multirow[t]{3}{*}{ CK (u.L) } & $A$ & $189.1(116.2)$ & $229.2(126.5)$ & 419.1 (194.8) & $278.4(120.5)$ & $195.0(79.3)$ & $216.3(153.5)$ \\
\hline & B & $311.1(200.8)$ & $372.0(189.2)$ & $627.3(451.1)$ & $457.6(401.6)$ & $430.1(577.2)$ & $401.4(538.9)$ \\
\hline & $\mathrm{C}$ & $182.3(123.0)$ & $232.6(124.1)$ & $450.4(286.6)$ & $266.4(120.5)$ & $204.9(110.1)$ & $164.8(69.0)$ \\
\hline \multirow[t]{3}{*}{ IL-6 (pg/ml) } & $A$ & $7951.1(3684.3)$ & 8195.7 (3812.0) & $8179.3(3680.1)$ & $8103.3(3794.3)$ & 8059.8 (3553.9) & $8092.4(3559.9)$ \\
\hline & B & $5528.0(2882.5)$ & $5534.2(2533.9)$ & $5751.6(2944.1)$ & $5528.0(2315.7)$ & $5254.7(2139.4)$ & $5652.2(2600.2)$ \\
\hline & C & $6267.1(2525.2)$ & 5913.1 (1766.5) & $5472.0(2216.7)$ & 5484.5 (1912.8) & $5130.4(2070.4)$ & 5987.6 (2765.9) \\
\hline
\end{tabular}


Fig. 1 Squat (a) and countermovement (b) jump performance before and up to 96h after damaging exercise. *Denotes a significant group effect

Fig. 2 Isokinetic muscle strength at $60^{\circ} \mathrm{s}-1$ (a) and $180^{\circ} \mathrm{s}-1$ (b) before and up to $96 \mathrm{~h}$ after damaging exercise

Fig. 3 Perceived muscle soreness before and up to $96 \mathrm{~h}$ after damaging exercise 
FIGURE 1

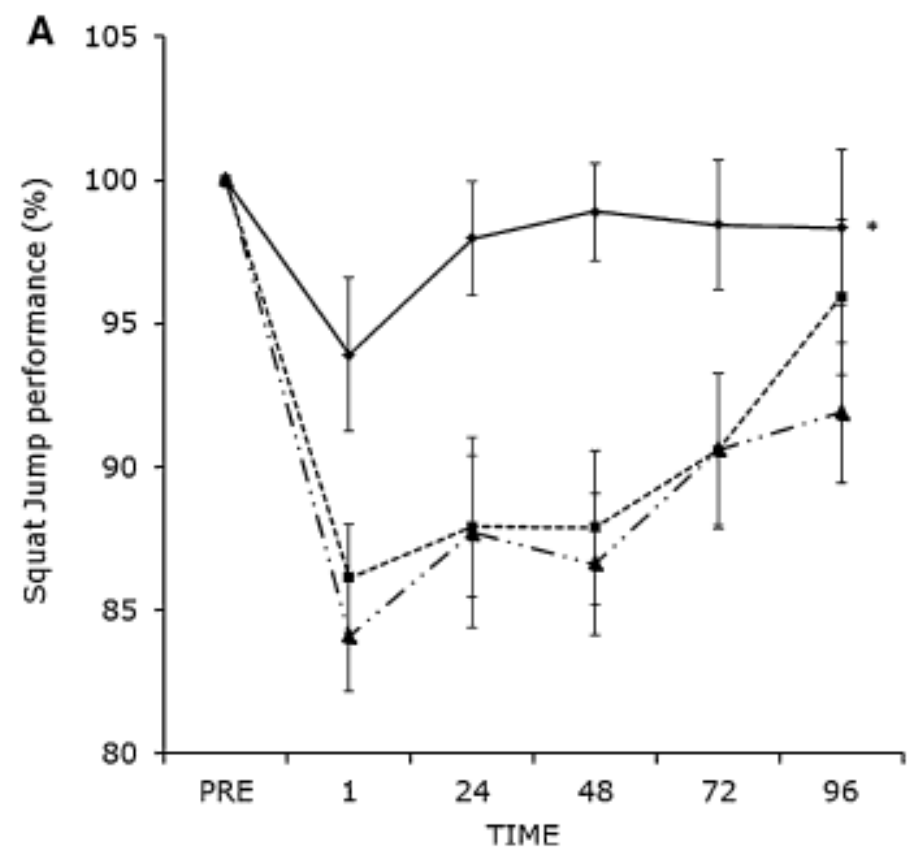

$\rightarrow$ High $\rightarrow$ Con $\rightarrow$ Low

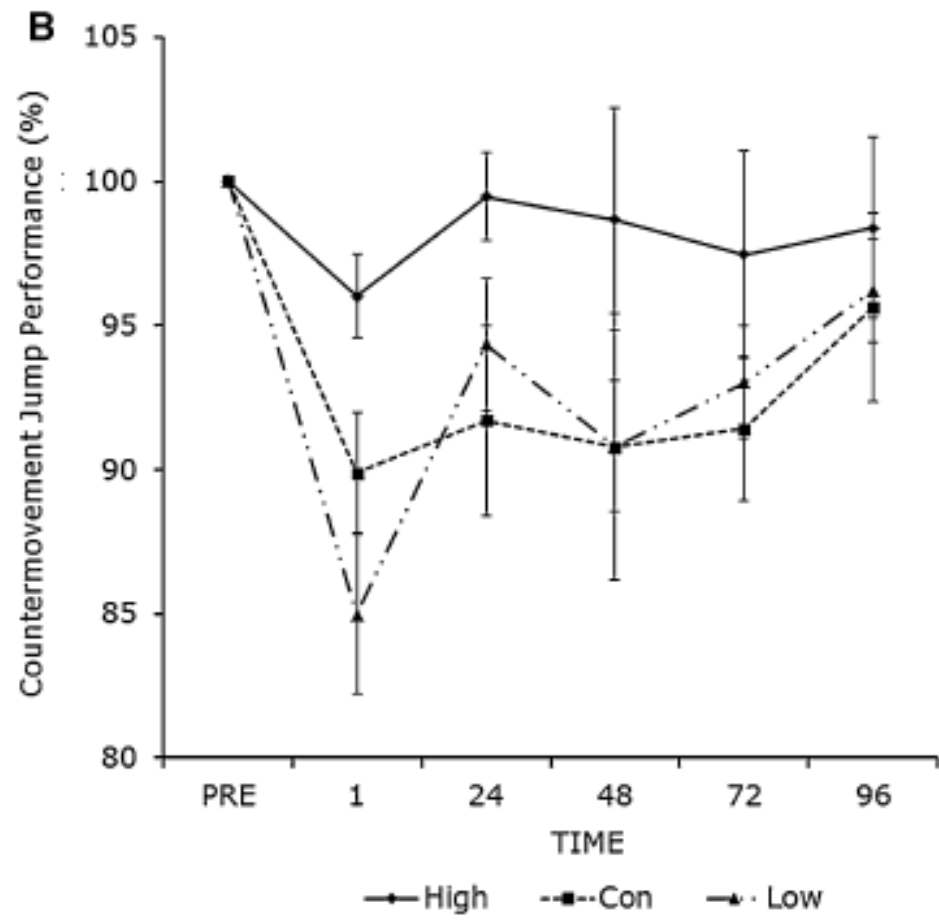


FIGURE 2

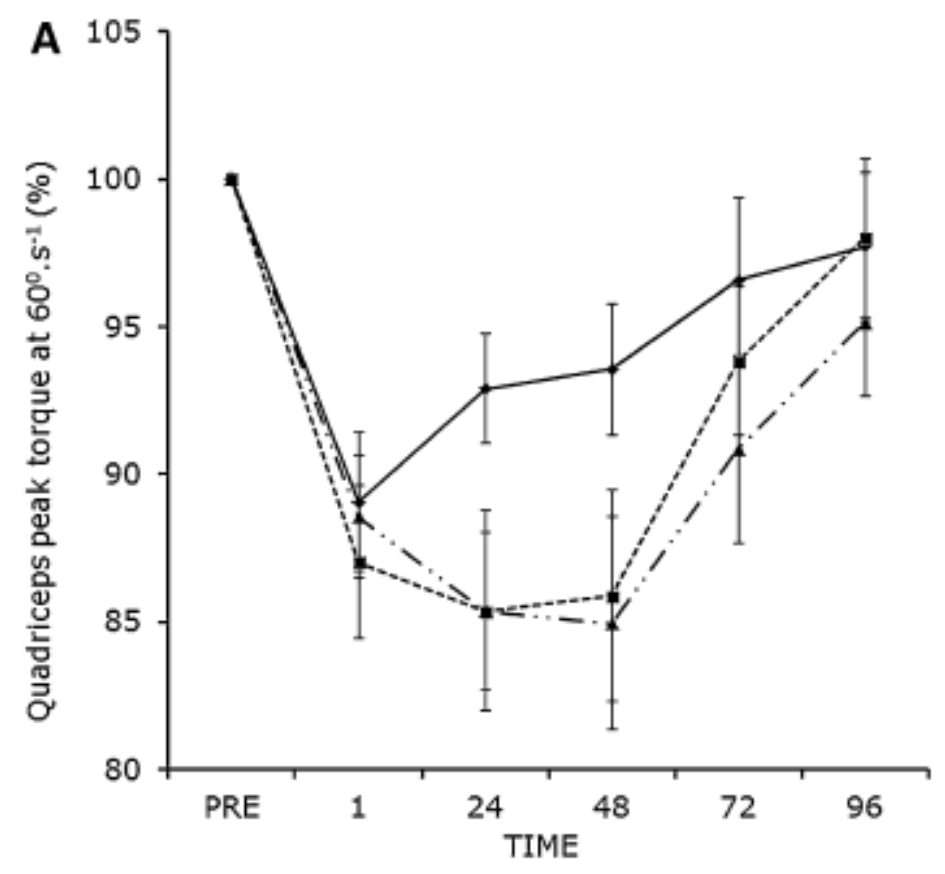

$\rightarrow$ High $\rightarrow$ Con $\rightarrow$ Low

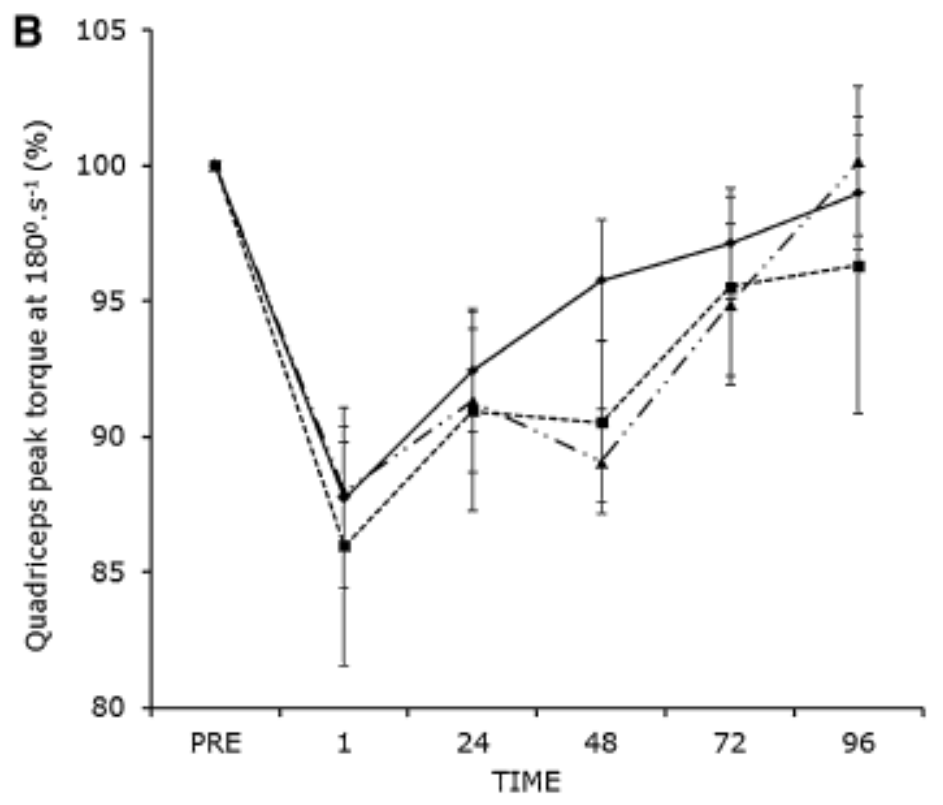

$\rightarrow$ High $\rightarrow-$ Con $\rightarrow$ Low 
FIGURE 3

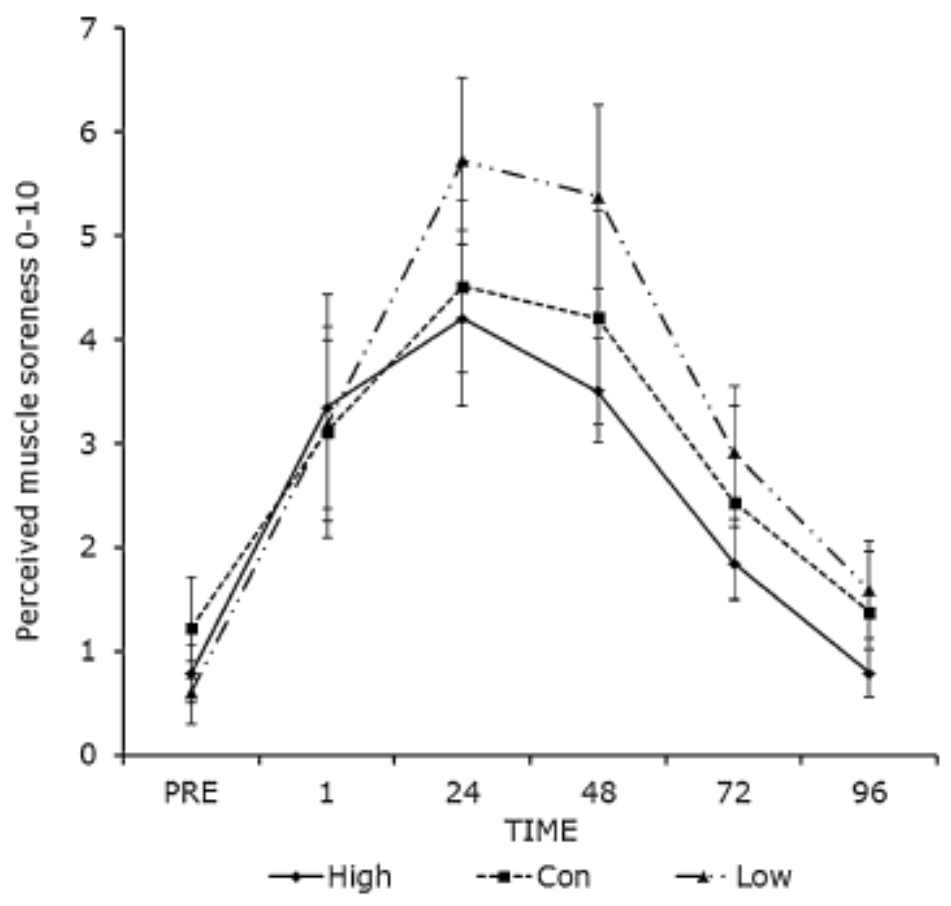

